\title{
ORGANIZACIÓN Y ACTIVIDADES PREVENTIVAS EN EL SECTOR DEL TRANSPORTE DE MERCANCÍAS POR CARRETERA ${ }^{(1)}$
}

\author{
ELENA ORDAZ CASTILLO*, ANGEL ASÚNSOLO DEL BARCO**, \\ JERÓNIMO MAQUEDA BLASCO*, AGUSTÍN SILVA MATO**, DAVID PRIETO MERINO** \\ (*) Escuela Nacional de Medicina del Trabajo. Instituto de Salud Carlos III \\ ${ }^{(* *)}$ Dpto. de Ciencias Sanitarias y Médico Sociales, Universidad de Alcalá de Henares
}

(1) Este artículo se desarrolla a partir de la Encuesta de Salud y Condiciones de Trabajo en el sector del transporte de mercancías por carretera , llevada a cabo por Escuela Nacional de Medicina del Trabajo (ISCIII) con la financiación de la Dirección General de transporte del Ministerio de Fomento.

\section{RESUMEN}

Objetivos: Identificar los recursos y actividades preventivas más prevalentes en el sector del transporte de mercancías por carretera y los hechos diferenciales con el resto de la población trabajadora española.

Metodología: Estudio descriptivo de los datos de la Encuesta de Salud y Condiciones de Trabajo del sector del transporte y estudio comparativo con el resto de la población trabajadora a través de la V-Encuesta Nacional de Condiciones de Trabajo. Para el análisis estadístico se ha aplicado el ji cuadrado y la t-student.

Resultados: E1 77\% de las empresas de este sector tienen menos de 50 trabajadores. El $41 \%$ de los conductores señala que en su empresa no existe Delegado de Prevención y un $25 \%$ no dispone de ningún Recurso Preventivo. Destaca el alto desconocimiento existente entre los conductores de los recursos preventivos en su empresa $(34,3 \%)$. La modalidad preventiva más frecuentemente instaurada en el sector es la asunción de la prevención por parte del empresario $(13,6 \%)$ a diferencia del resto de sectores donde predomina el SP. Ajeno (43\%).

Conclusiones: Predominio de la pequeña empresa en el Sector del transporte asociado a un escaso desarrollo del tejido preventivo en comparación con la población de referencia. Ambas características pueden ser determinantes de la menor actividad preventiva, más baja que la observada en la población de referencia. El acceso a actividades preventivas entre los conductores se relaciona con una mejor salud percibida.

\section{PALABRAS CLAVES}

Conductores, Transporte de mercancías por carretera, Organización preventiva, Recursos preventivos, Actividades preventivas, Salud percibida.

\section{ABSTRACT}

Goals: To identify the most prevalent resources and preventive activities in professional long-haul drivers and their peculiarities related to the other Spanish production branches.

Methodology: We develop a descriptive analysis of data from the Survey of Health and Working Conditions in professional truck drivers and we compare these results with the other Spanish production branches through the VNational Survey on Working Conditions. The statistical analysis applied T-Student test and the Ji square.

Results: In this branch $77 \%$ of companies have less than 50 employees. $41 \%$ of drivers referred that it does not exist the "Delegado de Prevención" (workers representative) in their companies and $25 \%$ that there is not any Preventive Action. It is specially relevant the unknowledge among drivers about preventive resources in their companies $(34.3 \%)$. The most frequently introduced preventive modality in the sector is the employer taking prevention by himself (13.6\%) whilst other sectors are mainly covered by External Preventive Services (43\%).

Conclusions: If Transport branch is compared with the general - reference population it shows an image in which undersized companies linked to a weak development of preventive network could be determinant to their lowest preventive activities. Also we find a better self perceived health related to a better access to preventive activities.

\section{KEY WORDS}

Long-haul drivers, Preventive Organization, Preventive Resources, Preventive activities, Self-perceived health. 


\section{A. SILVA MATO, D. PRIETO MERINO}

\section{INTRODUCCIÓN}

El objetivo de la prevención de riesgos laborales es proteger al trabajador de los riesgos que se derivan de su trabajo. Llevar a cabo una buena actuación en prevención de riesgos laborales implica la reducción de los riesgos, minimizando las causas de los accidentes y de las enfermedades profesionales y derivadas del trabajo.

Con la entrada en vigor de la Ley de Prevención de Riesgos Laborales (LPRL), se integra en la empresa la actividad preventiva a través de la obligación del empresario de adoptar una serie de medidas en materia de: evaluación de riesgos, información, consulta y participación de los trabajadores, formación, actuación en casos de emergencia y vigilancia de la salud. ${ }^{(1)}$

El cumplimiento de las obligaciones en materia de seguridad y salud en el trabajo, así como la gestión del conjunto de actividades relacionadas con la función preventiva se asegura mediante la organización de los recursos humanos y materiales adecuados a los riesgos de la empresa distinguiendo entre órganos de participación (delegado de prevención obligatorio en centros de trabajo de entre 6 y 50 trabajadores y comité de Seguridad y Salud obligatorio en las empresas o centros que cuenten con $50 \mathrm{o}$ más trabajadores), y una organización preventiva para afrontar las obligaciones preventivas del empresario, de acuerdo con el art. 10 del Reglamento de los Servicios de Prevención (SP), vinculada, principalmente, con la actividad de la empresa y el número de trabajadores. ${ }^{(2)}$

Tras la entrada en vigor de la LPRL y el Reglamento de los SP, los aspectos relativos a la modalidad preventiva adoptadas por las empresas han sido objeto de monitorización en las diferentes encuestas de ámbito laboral, como la Encuesta de Coyuntura Laboral del Ministerio de Trabajo y Asuntos Sociales de $1999^{\text {(3) }}$ o la Encuesta Nacional de Condiciones de Trabajo del Instituto de Seguridad e Higiene en el Trabajo (INSHT), incluyéndose además, en el parte de notificación de accidentes de trabajo, según la ORDEN TAS/2926/2002 de 19 de noviembre ${ }^{(4)}$.

El carácter longitudinal de las sucesivas Encuestas Nacionales de Condiciones de Trabajo del INSHT, permite analizar la tendencia en la implan- tación de los distintos modelos y recursos preventivos así como la evolución en el cumplimiento de la LPRL en materia de actividades preventivas.

En el año $2003^{(5)}$ la figura de Delegado de Prevención estaba presente en el $54,7 \%$ de los centros de trabajo de 6 ó más trabajadores, doce puntos de porcentaje por encima respecto al año $1999^{(6)}$ y veintiséis más respecto a $1997^{(7)}$. Para ese mismo año también se observa un incremento en el porcentaje de trabajadores que señalan que en su empresa o centro de trabajo existe un Comité de Seguridad y Salud, siendo de un 88\% mientras que en 1999 fué de un 85\%.

En general, respecto a 1999, aumentó la presencia de todas las modalidades de organización preventiva a excepción de la prevención asumida por el propio empresario ( $12 \%$ en 2003 y $28 \%$ en 1999), probablemente debido a la asunción de recursos preventivos más profesionalizados.

En consonancia con estos resultados, el análisis de las sucesivas Encuestas Nacionales de Condiciones de Trabajo revela una tendencia creciente en la frecuencia de actividades preventivas.

La organización y el desarrollo de actividades preventivas, así como su eficacia cobran especial interés en actividades tales como el transporte de mercancías por carretera que constituye una actividad clave para el desarrollo productivo de nuestro país. La alta siniestralidad de este sector junto con los numerosos problemas de salud asociados a las peculiares condiciones de trabajo que soportan $(8,9,10,11)$, hace de este colectivo un sector vulnerable donde la organización preventiva se impone como una solución para reducir los riesgos.

Este estudio persigue ampliar el conocimiento sobre la estructura y organización preventiva en este sector y su influencia en las actividades de prevención de riesgos laborales y su repercusión sobre la salud de los trabajadores, bajo la hipótesis que una buena organización preventiva contribuye no sólo a reducir la siniestralidad laboral en la empresa, si no también, a conseguir un ambiente de trabajo más saludable y a mejorar la calidad de vida laboral. Así mismo, trata de identificar los hechos diferenciales de la organización preventiva del sector con respecto a la organización preventiva del conjunto de actividades económicas del país. 


\section{MATERIAL Y MÉTODOS}

A partir de la Encuesta de Salud y Condiciones de trabajo (ESCT) en el transporte de mercancías por carretera $^{(12)}$, se han seleccionado las variables relacionadas con la organización de las empresas, actividades en materia de prevención de riesgos laborales y con la percepción del estado de salud de los trabajadores.

De esta forma se analizaron variables relativas a:

1. Las características de los trabajadores y de las empresas del sector: "Tamaño de la empresa", "situación laboral" y "antigüedad laboral y en la empresa".

2. La organización preventiva: Entendiendo por tal los órganos de participación (conocimiento de las figuras de "representante legal de los trabajadores", y "delegado de prevención") y las "modalidades preventivas".

3. Las actividades preventivas: "Reconocimientos médicos", "información" y "formación" recibida por los conductores.

\section{Salud percibida de los trabajadores.}

Se realizó un análisis descriptivo de estas variables y, a continuación, se comparó, mediante análisis bivariante, las posibles relaciones que podrían encontrarse entre ellas.

El análisis comparativo de la organización preventiva en el sector del Transporte con el resto de la población trabajadora de nuestro país se realizó a través de la V Encuesta Nacional de Condiciones de Trabajo del Instituto Nacional de Seguridad e Higiene en el Trabajo del Ministerio de Trabajo y Asuntos Sociales, (V ENCT). ${ }^{(5)}$

Para garantizar la comparabilidad entre nuestro estudio y la VENCT, se seleccionó una sub-muestra, de tal manera que el grupo de comparación tuviera una estructura por edad, sexo y situación de alta laboral comparable a la muestra de conductores de nuestro estudio, eliminando de ella a los trabajadores que realizaban su actividad en el sector del transporte. La población resultante de esta submuestra fue de 3516 trabajadores.

Se comparó el conocimiento de la figura del delegado de prevención, la modalidad preventiva establecida y las actividades preventivas realizadas. En este último caso, se analizaron los reconocimientos médicos periódicos y la formación recibida ya que, el resto de variables (información sobre los riesgos de la carga, reconocimientos para renovar el carnet de conducir, etc), no eran comunes a las dos encuestas.

La estimación de prevalencias de las distintas variables estudiadas se realizó con intervalos de confianza al 95\%. Para la comparación de proporciones se utilizó el Ji cuadrado y para la comparación de medias la t-student.

\section{RESULTADOS}

El 77,1\% de los trabajadores del sector del transporte de mercancías por carretera son trabajadores por cuenta ajena de los cuales el $61,9 \%$ tiene un contrato indefinido (tabla 1).

Tabla 1: Situación laboral del sector del transporte

\begin{tabular}{rcccc}
\hline & $\mathrm{n}$ & $\%$ & \multicolumn{2}{c}{ IC al 95\% } \\
\hline Autónomo & 258 & 22,9 & 20,3 & 25,3 \\
Asalariados & 871 & 77,1 & 74,6 & 79,6 \\
\hline Indefinido & 699 & 61,9 & 59 & 64,8 \\
Por obra o servicio & 82 & 7,3 & 5,7 & 8,8 \\
Eventual & 55 & 4,9 & 3,5 & 6,2 \\
Interino & 6 & 0,5 & 0,06 & 1 \\
De formación & 1 & 0,1 & 0,002 & 0,5 \\
ETT & 6 & 0,5 & 0,06 & 1 \\
Otro & 17 & 1,5 & 0,7 & 2,2 \\
Nc & 6 & 0,5 & 0,069 & 1 \\
\hline
\end{tabular}

Base: total de conductores ( ESCT en el Sector del Transporte) 


\section{A. SILVA MATO, D. PRIETO MERINO}

El análisis comparativo de la antigüedad en la profesión, revela diferencias estadísticamente significativas entre este sector y el resto de sectores de actividad representado por la población de referencia (tabla 2). Se observa una concentración de la vida laboral del conductor en el grupo de 6 a 20 años de antigüedad (55\% frente al $46,9 \%$ del resto de sectores de actividad), y una menor presencia de los mismos en los intervalos de 0 a 5 años de antigüedad $(21,8 \%$ frente a un $25,8 \%)$ y de más de 20 años de antigüedad $(23,2 \%$ frente a un $27,4 \%)$.
En cuanto a la antigüedad en la empresa (tabla 3), la población de conductores se caracteriza por una mayor presencia de trabajadores con una antigüedad menor a un año (16 puntos de porcentaje de diferencia respecto a la población de referencia) y una menor presencia de trabajadores con una antigüedad superior a los 10 años $(-25,9$ puntos $\%$ de diferencia), diferencias que resultaron ser estadísticamente significativas.

Tabla 2: Distribución de las poblaciones de estudio según antigüedad en la profesión (\%)

\begin{tabular}{|c|c|c|c|c|}
\hline & 0 a 5 años & 6 a 20 años & $>20$ años & p-val \\
\hline Submuestra V ENCT (no conductores) & $25,6 \%$ & $46,9 \%$ & $27,4 \%$ & 0,000 \\
\hline Conductores & $21,8 \%$ & $55,0 \%$ & $23,2 \%$ & \\
\hline
\end{tabular}

Tabla 3: Distribución de las poblaciones de estudio según antigüedad en la empresa (\%)

\begin{tabular}{lcccc}
\hline & $<1$ año & $1-10$ años & $>10$ años & p-val \\
\hline Submuestra V ENCT (no conductores) & $8,8 \%$ & $52,5 \%$ & $38,7 \%$ & \multirow{2}{*}{0,000} \\
Conductores & $24,9 \%$ & $62,3 \%$ & $12,8 \%$ & \\
\hline \multicolumn{1}{c}{ Base: ESCT en el Sector del Transporte; Submuestra V- ENCT } & &
\end{tabular}

En el análisis de la distribución de los centros de trabajo por tamaño de plantilla se observa que el $76,9 \%$ de las empresas de este sector están constituidas por menos de 50 trabajadores y cabe destacar que un $24,6 \%$ lo forman empresas de menos de seis trabajadores (tabla 4).
$\mathrm{Al}$ analizar la organización preventiva del sector y compararla con la población de referencia en trabajadores de empresas con seis o más empleados se observan diferencias estadísticamente significativas.

Tabla 4: Tamaño de plantilla de las empresas del sector del transporte

\begin{tabular}{lcccc}
\hline & $\mathrm{n}$ & $\%$ & \multicolumn{2}{c}{ IC al 95\% } \\
\hline$<6$ & 214 & 24,6 & 21,7 & 27,5 \\
$6-9$ & 126 & 14,5 & 12,1 & 16,9 \\
$10-49$ & 330 & 37,9 & 34,6 & 41,2 \\
$50-249$ & 121 & 13,9 & 11,5 & 16,2 \\
$\geq 250$ & 80 & 9,2 & 7,2 & 11,2 \\
Total & 871 & 100,0 & & \\
\hline \multicolumn{2}{l}{ Base: conductores asalariados (ESCT en el Sector del Transporte) }
\end{tabular}


El 34\% de los conductores conoce la existencia de delegado de prevención en su centro de trabajo frente al $77 \%$ en la población de referencia, destacando el porcentaje de conductores que manifiesta su desconocimiento sobre la existencia de esta figura en su centro de trabajo, que supera en más del doble a la población de referencia (Tabla 5).
Son los conductores de mayor antigüedad en la empresa y aquellos pertenecientes a empresas de gran tamaño quienes de forma significativa afirman conocer con una mayor frecuencia la existencia de esta figura (tabla 6).

Tabla 5: Conocimiento de la existencia de Delegado de Prevención en la empresa, según Actividad Profesional (\%)

\begin{tabular}{lcccccc}
\hline & $\begin{array}{c}\text { Submuestra VENCT } \\
\text { (no Conductores) }\end{array}$ & \multicolumn{1}{c}{ IC al 95\% } & $\begin{array}{c}\text { Conductores } \\
\text { (ESCT) }\end{array}$ & IC al 95\% \\
\hline NO & $12 \%$ & 10,9 & 13,1 & $41 \%$ & 37,2 & 45 \\
SI & $77 \%$ & 75,5 & 78,5 & $34 \%$ & 30,2 & 37,6 \\
Ns & $11 \%$ & 9,9 & 21,1 & $25 \%$ & 21,5 & 28,4 \\
Total & $100 \%$ & & & $100 \%$ & & \\
Base & 3.148 & & 637 & & \\
\hline p<0,001 & \\
Base: trabajadores asalariados de centros con seis o más trabajadores (ESCT en el Sector del \\
Transporte; Submuestra V- ENCT)
\end{tabular}

\section{Tabla 6: Relación entre Antigüedad (en años) en la empresa actual del conductor y tamaño de plantilla según el conocimiento de la existencia de Delegado de Prevención (\%)}

\begin{tabular}{lcccc}
\hline $\begin{array}{l}\text { Conoce la figura del delegado de } \\
\text { prevención }\end{array}$ & $\mathrm{N}$ & Media & Desviación típica & p-val \\
\hline Antigüedad (en años) en la empresa actual & & & & \\
\hline $\mathrm{Si}$ & 211 & 5,97 & 6,3 & 0,000 \\
$\mathrm{No}$ & 398 & 4,35 & 5,59 & \\
\hline Tamaño de plantilla & & & & 0,000 \\
\hline $\mathrm{Si}$ & 211 & 136,58 & 262,89 & \\
$\mathrm{No}$ & 399 & 52,04 & 138,13 &
\end{tabular}

También se observa un desconocimiento sobre las modalidades preventivas implantadas en el centro de trabajo por parte del conductor $(34,3 \%)$ respecto a la población de referencia $(16 \%)$. En este caso, uno de cada cinco conductores afirmó que no existía ningún modelo de prevención frente al 3,2\% del resto de trabajadores (tabla 7).

En el sector del transporte predomina la asunción por parte del empresario de la organización preven- tiva $(13,6 \%)$ seguida del Servicio de Prevención Ajeno (SPA) (10\%) mientras que, en la población de referencia, las modalidades preventivas de mayor prevalencia son los SPA (43\%), los servicios de prevención propios (SPP) $(34,8 \%)$ y los trabajadores de la empresa designados por parte del empresario $(25,8 \%)$.

El SPP es el recurso preventivo menos frecuente entre los conductores y se relaciona con el tamaño de 
Tabla 7: Conocimiento de la existencia de la modalidad preventiva en la empresa, según Actividad Profesional (\%)

\begin{tabular}{lccc}
\hline & $\begin{array}{c}\text { Submuestra VENCT } \\
\text { (no Conductores) }\end{array}$ & Conductores & p-val \\
\hline SP Propio & $34,8 \%$ & $5,0 \%$ & 0,000 \\
SP Ajeno & $\mathbf{4 3 , 0} \%$ & $10,2 \%$ & 0,000 \\
Trabajador Designado & $25,5 \%$ & $8,5 \%$ & 0,000 \\
Asunción por el Empresario & $5,7 \%$ & $13,6 \%$ & 0,000 \\
No Sabe & $16,0 \%$ & $34,3 \%$ & 0,000 \\
Ninguno & $3,2 \%$ & $24,5 \%$ & 0,000 \\
No contesta & $0,5 \%$ & $5,4 \%$ & 0,000 \\
Base & 3.516 & 1.129 & \\
\hline Base: Total de trabajadores de la ESCT en el Sector del Transporte y la Submuestra & V- ENCT \\
En negrita se indican las mayores prevalencias por grupo profesional &
\end{tabular}

plantilla de tal manera que la probabilidad de tener implantado un $S P P$ es significativamente mayor en grandes empresas. Los conductores de empresas pequeñas manifiestan más frecuentemente que en su centro no existe SP (tabla 8).

En la tabla 9 se observa que a medida que aumenta el tamaño de plantilla se produce una disminución en el porcentaje de conductores que refieren que en su empresa no existe modalidad preventiva y el SPP se hace más presente en empresas de más de 250 trabajadores.

En general, los conductores disponen de manera estadísticamente significativa de menos cobertura por actividades de prevención de riesgos laborales que la población de referencia (tabla 10). El 23\% de conductores reconoce haberse sometido a reconocimiento médico periódico relativo a riesgos laborales en el último año frente al $71 \%$ de la población de referencia, y un $21,0 \%$ de los conductores habían recibido formación en el último año frente al $60,7 \%$ del resto de trabajadores. En cuanto al tipo de formación en los conductores predomina la destinada a prevenir riesgos laborales $(12,9 \%)$ frente a mejorar el trabajo $(8,8 \%)$.

En la tabla 11 se observa que el desarrollo de las diferentes actividades preventivas en ambos colectivos profesionales se da con una mayor frecuencia cuando existe en la empresa la figura del Delegado de Prevención o, al menos, el trabajador manifiesta conocer su existencia.

Tabla 8: Relación entre tamaño de plantilla y Modalidad Preventiva (SPP o ninguna) en el centro de trabajo

\begin{tabular}{lccccc}
\hline & $N$ & Media & Desviación típica. & p-val \\
\hline SPP & Sí & 46 & 156,48 & 242,39 & \\
\hline & No & 793 & 54,96 & 162,91 & 0,000 \\
& Sí & 179 & 27,69 & & \\
\hline Ninguna & No & 660 & 69,43 & 59,95 & 0,000 \\
& & & 187,74 & \\
\hline \multicolumn{2}{l}{ Base: Total de conductores (ESCT en el Sector del Transporte) } &
\end{tabular}


Tabla 9: Modalidad preventiva según tamaño del centro trabajo en el Sector del transporte (\%)

\begin{tabular}{lcccc}
\hline \multicolumn{5}{c}{ plantilla (en rango) del centro de trabajo } \\
\hline & $6-10$ & $10-49$ & $50-249$ & $>\mathbf{2 5 0}$ \\
Trab. designado & $6,3 \%$ & $10,0 \%$ & $\mathbf{1 1 , 6 \%}$ & $10,0 \%$ \\
SPP & $3,2 \%$ & $5,8 \%$ & $5,0 \%$ & $\mathbf{1 6 , 3 \%}$ \\
SPA & $13,5 \%$ & $\mathbf{1 0 , 3 \%}$ & $10,7 \%$ & $11,3 \%$ \\
Empresario & $7,9 \%$ & $8,8 \%$ & $12,4 \%$ & $13,8 \%$ \\
Ninguno & $22,2 \%$ & $22,4 \%$ & $17,4 \%$ & $7,5 \%$ \\
Ns & $39,7 \%$ & $42,1 \%$ & $41,3 \%$ & $45,0 \%$ \\
Nc & $7,1 \%$ & $3,3 \%$ & $2,5 \%$ & $3,8 \%$ \\
\hline Total & 126 & 330 & 121 & 80 \\
\hline
\end{tabular}

Base: conductores asalariados (ESCT en el Sector del Transporte)

En negrita se indican las mayores prevalencias por tamaño de centro de trabajo

Tabla 10: Actividades preventivas según Actividad Profesional (\%)

\begin{tabular}{lccc}
\hline & $\begin{array}{c}\text { Submuetra VENCT } \\
\text { (no Conductores) }\end{array}$ & Conductores & p-val \\
\hline Reconocimiento Médico Periódico & $71,0 \%$ & $23,0 \%$ & 0.0000 \\
Formación en último año & $60,7 \%$ & $21,0 \%$ & 0.0000 \\
Formación para mejorar trabajo & $34,2 \%$ & $8,8 \%$ & 0.0000 \\
Formación para riesgos laborales & $37,9 \%$ & $12,9 \%$ & 0.0000 \\
Base & 3.516 & 1.129 & \\
\hline
\end{tabular}

Base: ESCT en el Sector del Transporte; Submuestra V- ENCT

Tabla 11: Relación entre conocimiento de la existencia de Delegado de Prevención y Actividades Preventivas según Actividad Profesional (\%)

\begin{tabular}{lcccccccc}
\hline & $\begin{array}{c}\text { Reconocimientos } \\
\text { médicos periódicos }\end{array}$ & \multicolumn{2}{c}{$\begin{array}{c}\text { Formación en el } \\
\text { último año }\end{array}$} & \multicolumn{2}{c}{$\begin{array}{c}\text { Formación para } \\
\text { mejorar el trabajo }\end{array}$} & \multicolumn{2}{c}{$\begin{array}{c}\text { Formación sobre } \\
\text { riesgos laborales }\end{array}$} \\
\hline & V ENCT & CONDUC & VENCT & CONDUC & V ENCT & CONDUC & VENCT & CONDUC \\
\hline No & $51 \%$ & $20 \%$ & $43 \%$ & $13 \%$ & $28 \%$ & $5 \%$ & $18 \%$ & $7 \%$ \\
SI & $80 \%$ & $36 \%$ & $69 \%$ & $42 \%$ & $39 \%$ & $20 \%$ & $45 \%$ & $29 \%$ \\
Ns & $59 \%$ & $21 \%$ & $45 \%$ & $16 \%$ & $28 \%$ & $6 \%$ & $24 \%$ & $10 \%$ \\
Nc & $49 \%$ & $20 \%$ & $40 \%$ & $17 \%$ & $17 \%$ & $6 \%$ & $25 \%$ & $9 \%$ \\
p-val & 0.00 & 0.000 & 0.000 & 0.000 & 0.000 & 0.000 & 0.000 & 0.000 \\
\hline
\end{tabular}

Base: ESCT en el Sector del Transporte; Submuestra V- ENCT

En cuanto a las modalidades preventivas (tabla 12), su inexistencia o desconocimiento se asocia en ambos colectivos profesionales con peores resultados en términos de actividad preventiva.

En general, los mejores indicadores de actividad preventiva se relacionan con tener implantado un $S P P$ mientras que los porcentajes más bajos de actividad preventiva se dan cuando es el propio empresario quien asume las tareas de prevención.
Atendiendo a la situación laboral de los conductores se observa que, en asalariados predominan actividades preventivas tales como la formación y la vigilancia médica mientras que, son los autónomos quienes reflejan los porcentajes más elevados en cuanto a la información recibida, siendo la información recibida sobre medidas preventivas en el centro de destino estadísticamente superior a los asalariados (tabla 13). 
Tabla 12: Relación entre actividades preventivas y modalidad preventivas, según Actividad profesional (\%)

\begin{tabular}{|c|c|c|c|c|c|c|c|c|}
\hline & \multicolumn{2}{|c|}{$\begin{array}{l}\text { Reconocimientos } \\
\text { médicos periódicos }\end{array}$} & \multicolumn{2}{|c|}{$\begin{array}{l}\text { Formación en el } \\
\text { último año }\end{array}$} & \multicolumn{2}{|c|}{$\begin{array}{l}\text { Formación para } \\
\text { mejorar el trabajo }\end{array}$} & \multicolumn{2}{|c|}{$\begin{array}{l}\text { Formación sobre } \\
\text { riesgos laborales }\end{array}$} \\
\hline & VENCT & CONDUCT & VENCT & CONDUCT & VENCT & CONDUCT & VENCT & CONDUCT \\
\hline Trab.designado & $79 \% *$ & $45 \% *$ & $67 \% *$ & $39 \% *$ & $36 \% *$ & $16 \% *$ & $44 \% *$ & $28 \%{ }^{*}$ \\
\hline Sp propio & $79 \% *$ & $45 \% *$ & $77 \% *$ & $37 \% *$ & $47 \% *$ & $23 \% *$ & $52 \% *$ & $25 \%$ \\
\hline SP. ajeno & $73 \% *$ & $25 \%$ & $61 \%$ & $40 \% *$ & $32 \% *$ & $15 \% *$ & $39 \%$ & $26 \% *$ \\
\hline Empresario & $63 \% *$ & $26 \%$ & $51 \% *$ & $23 \%$ & $30 \%$ & $12 \%$ & $37 \%$ & $16 \%$ \\
\hline Ninguno & $43 \% *$ & $18 \% *$ & $34 \% *$ & $10 \% *$ & $23 \% *$ & $3 \% *$ & $10 \% *$ & $5 \% *$ \\
\hline Ns & $60 \% *$ & $19 \%$ * & $45 \% *$ & $15 \% *$ & $26 \% *$ & $7 \% *$ & $25 \%$ * & $8 \% *$ \\
\hline $\mathrm{Nc}$ & $75 \%$ & $15 \%$ & $47 \%$ & $25 \%$ & $33 \%$ & $14 \%$ & $27 \%$ & $15 \%$ \\
\hline
\end{tabular}

Base: ESCT en el Sector del Transporte; Submuestra V- ENCT

$\left(^{*}\right)$ indica diferencias estadísticamente significativas entre quienes indican tener recurso preventivo implantado y los que no

Tabla 13: Distribución de Actividades Preventivas en el Sector del Transporte según situación laboral (\%)

\begin{tabular}{lccccc}
\hline & Formación & $\begin{array}{c}\text { Información } \\
\text { riesgos de la } \\
\text { carga }\end{array}$ & $\begin{array}{c}\text { Información } \\
\text { protección } \\
\text { prevención }\end{array}$ & $\begin{array}{c}\text { Información } \\
\text { centros de } \\
\text { destino }\end{array}$ & $\begin{array}{c}\text { Vigilancia } \\
\text { médica }\end{array}$ \\
\hline Asalariado & $\mathbf{2 2 , 6 \% *}$ & $43,5 \%$ & $\mathbf{4 2 , 4 \%}$ & $39,0 \%$ & $\mathbf{5 3 , 2} \%$ \\
Autónomo & $15,4 \%$ & $\mathbf{4 9 , 6 \%}$ & $\mathbf{4 8 , 1} \%$ & $\mathbf{5 9 , 7 \% ^ { * }}$ & $44,6 \%$ \\
\hline
\end{tabular}

Los \% se expresan frente al total de trabajadores por situación laboral

Base: total de conductores (ESCT en el Sector del Transporte)

En negrita se indican las mayores prevalencias

$\left.{ }^{*}\right)$ refleja diferencias estadísticamente significativas.

En el análisis de la influencia de las actividades preventivas sobre la percepción del estado de salud de los conductores se observa, en líneas generales, una relación positiva.

Los conductores que reconocen haberse realizado a un reconocimiento médico en el último año, manifiestan de manera estadísticamente significativa mejores indicadores de salud percibida (Tabla 14).
Lo mismo ocurre con aquellos conductores que han recibido información en su centro de trabajo sobre los riesgos de la carga que transportan (Tabla 15).

En cuanto a la formación recibida (charla, curso o material didáctico) de cualquier tema facilitado por su asociación, sindicato o empresa en el último año no se observa relación estadísticamente significativa con el estado de salud percibido (Tabla 16).

\section{Tabla 14: Relación entre haberse realizado un reconocimiento médico y salud percibida}

\begin{tabular}{lcccccc}
\hline Reconocimiento & \multicolumn{7}{c}{ Percepción del estado de salud (\%) } \\
\cline { 2 - 7 } médico & Muy bueno & Bueno & Regular & Malo & Muy malo & Total \\
$\mathrm{Si}$ & $26,0 \%$ & $54,2 \%$ & $17,5 \%$ & $1,6 \%$ &, $7 \%$ & $100,0 \%$ \\
$\mathrm{No}$ & $17,4 \%$ & $58,4 \%$ & $21,4 \%$ & $2,6 \%$ &, $2 \%$ & $100,0 \%$ \\
Total & $21,6 \%$ & $56,3 \%$ & $19,5 \%$ & $2,1 \%$ &, $4 \%$ & $100,0 \%$ \\
\hline Base & 244 & 635 & 220 & 24 & 5 & 1128 \\
\hline
\end{tabular}


Tabla 15: Relación entre haber recibido información sobre los riesgos específicos de la carga y salud percibida

\begin{tabular}{lcccccc}
\hline Información & \multicolumn{7}{c}{ Percepción del estado de salud (\%) } \\
\cline { 2 - 7 } & Muy bueno & Bueno & Regular & Malo & Muy malo & Total \\
\hline $\mathrm{Si}$ & $25,5 \%$ & $54,1 \%$ & $18,8 \%$ & $1,2 \%$ &, $4 \%$ & $100,0 \%$ \\
$\mathrm{No}$ & $17,6 \%$ & $58,6 \%$ & $20,5 \%$ & $2,9 \%$ &, $5 \%$ & $100,0 \%$ \\
Total & $21,1 \%$ & $56,6 \%$ & $19,7 \%$ & $2,2 \%$ &, $5 \%$ & $100,0 \%$ \\
Base & 234 & 629 & 219 & 24 & 5 & 1111 \\
\hline $\mathrm{P}=0,003$ & Base: conductores ESCT en el Sector del Transporte
\end{tabular}

Tabla 16: Relación entre formación recibida y salud percibida en el
Sector del Transporte

\begin{tabular}{|c|c|c|c|c|c|c|}
\hline \multirow{3}{*}{$\begin{array}{l}\text { Formación } \\
\text { recibida en } \\
\text { el último año } \\
\mathrm{Si} \\
\text { No }\end{array}$} & \multicolumn{6}{|c|}{ Percepción del estado de salud (\%) } \\
\hline & Muy bueno & Bueno & Regular & Malo & Muy malo & Total \\
\hline & $\begin{array}{l}23,6 \% \\
21,2 \%\end{array}$ & $\begin{array}{l}56,7 \% \\
56,6 \%\end{array}$ & $\begin{array}{l}18,9 \% \\
19,3 \%\end{array}$ & $\begin{array}{r}, 4 \% \\
2,5 \%\end{array}$ & $\begin{array}{l}, 4 \% \\
, 5 \%\end{array}$ & $\begin{array}{l}100,0 \% \\
100,0 \%\end{array}$ \\
\hline Total & $21,7 \%$ & $56,6 \%$ & $19,2 \%$ & $2,1 \%$ &, $5 \%$ & $100,0 \%$ \\
\hline Base & 241 & 628 & 213 & 23 & 5 & 1110 \\
\hline
\end{tabular}

\section{CONCLUSIONES}

De acuerdo a los resultados del estudio, la actividad del transporte de mercancías por carretera, largo recorrido, se realiza por, casi un $25 \%$ de trabajadores autónomos y por un tejido empresarial caracterizado por el predominio de la muy pequeña empresa (el 39\% de las misma cuentan con menos de 10 trabajadores y sólo un $9,2 \%$ lo constituyen empresas de más de 250 trabajadores).

La temporalidad es este sector se sitúa en torno al $15 \%$.

Tanto la antigüedad en la empresa como en la profesión es menor en la población de conductores respecto al resto de sectores de actividad, presentando prevalencias menores en los intervalos de mayor antigüedad en la profesión (>20 años) y en la empresa (> 10 años).

En relación a la participación en la empresa mediante la figura de Delegado de Prevención, la situación más frecuente es la "no existencia" de esta figura, expresado en un $41 \%$ de los casos, situación claramente diferenciada con el resto de actividades económicas en las que un $77 \%$ de los casos responde disponer de Delegado de Prevención.

El "desconocimiento" de la existencia de Delegado de Prevención es, también, significativamente más bajo en el sector del transporte de mercancías por carretera $(25 \%)$ que en la población tomada de referencia $(11 \%)$.

En lo que se refiere a la modalidad preventiva, de nuevo se señala como la situación más frecuente por parte de los conductores el "desconocimiento" de su existencia (39,3\%), seguida de la "no existencia" de ningún tipo de modalidad preventiva $(24,5 \%)$. Esta situación difiere notablemente de la población de referencia, donde la "no existencia" de ningún tipo de modalidad preventiva es de un 3,2\% y el "desconocimiento" de su existencia es del $16 \%$.

La modalidad preventiva más frecuentemente implantada en la población de referencia es el $S P P$ y el SPA mientras que en el sector del transporte predomina la asunción por parte del empresario de la actividad preventiva. 


\section{A. SILVA MATO, D. PRIETO MERINO}

En general, los conductores disponen de una menor cobertura por actividades de prevención de riesgos laborales que la población de referencia; el $23 \%$ de conductores se sometió a reconocimiento médico periódico relativo a riesgos laborales frente al $71 \%$ de la población de referencia y el $21 \%$ de los conductores recibió formación en el último año frente al $60,7 \%$ del resto de trabajadores.

Estas actividades preventivas son significativamente más frecuentes en conductores que responden conocer a los órganos de participación de su empresa y la modalidad preventiva implantada si bien varía en función del tipo de recurso implantado.

Para ambos colectivos profesionales, las actividades preventivas son más frecuentes en trabajadores de empresas donde existe Delegado de Prevención y modalidad preventiva implantada, fundamentalmente cuando existe un $S P P$, mientras que los indicadores más bajos de actividad preventiva se dan cuando es el propio empresario quien asume las tareas de prevención. En este punto conviene recordar que es, esta última modalidad preventiva, la más frecuente en el sector del transporte, posiblemente debido al predominio de la pequeña empresa.

En el análisis de la influencia de las actividades preventivas sobre la percepción del estado de salud de los conductores se observa, en líneas generales, una relación positiva de tal forma que haber recibido información sobre riesgos laborales y haberse sometido a vigilancia médica, mejora el nivel de salud percibida entre los conductores.

\section{DISCUSIÓN}

Los resultados del estudio en relación a la antigüedad en la profesión, ponen de manifiesto una vida laboral más breve del conductor con respecto a la población de referencia; cerca de una cuarta parte de conductores tiene una antigüedad en la empresa de menos de un año y un $12,8 \%$ supera los 10 años, fenómeno inverso al ocurrido en la población de referencia donde el colectivo de trabajadores con más de 10 años de antigüedad supera en 30 puntos porcentuales al colectivo de trabajadores de menos de un año de antigüedad.

Este resultado podría estar indicando una salida de los conductores hacia el mercado de autónomos o bien una mayor rotación de los mismos por diferentes empresas.

En nuestra opinión, existe una profunda diferencia en el desarrollo del tejido preventivo de las empresas del transporte con respecto a las empresas de otros sectores, tanto en lo que se refiere a la figura de Delegado de Prevención como a la adopción de una modalidad preventiva consolidada.

Este escaso desarrollo del tejido preventivo en las empresas puede ser un elemento determinante de los indicadores de actividad preventiva, más bajos que los presentados por la población de referencia.

Los resultados muestran la evidencia de una relación positiva entre las actividades preventivas estudiadas y una mejor percepción del estado de salud.

La movilidad del puesto de trabajo del conductor puede ser uno de los determinantes del desconocimiento que el conductor tiene de las estructuras preventivas existentes en la empresa, posibilidad que debe exigir un esfuerzo en la información de su existencia.

Tanto por las características de las empresas del sector (pequeña empresa) como por la naturaleza propia de la actividad de la conducción, la organización preventiva se evidencia como una clara oportunidad de mejora en este sector. 


\section{BIBLIOGRAFIA}

1. LEY $31 / 1995$, de 8 de noviembre de prevención de riesgos laborales. BOE $\mathrm{n}^{\circ} 269$, de 10 de noviembre

2. REAL DECRETO 39/1997, de 17 de enero, por el que se aprueba el Reglamento de los Servicios de Prevención. BOE núm. 27 de 31 enero.

3. Encuesta de Coyuntura Laboral. Anuario de Estadísticas Laborales y Asuntos Sociales 1999 del Ministerio de Trabajo y Asuntos Sociales 1999

4. ORDEN TAS/2926/2002, de 19 de noviembre, por la que se establecen nuevos modelos para la notificación de los accidentes de trabajo y se posibilita su transmisión por procedimiento electrónico. BOE núm. 279 de 21 de noviembre de 2002

5. V Encuesta Nacional de Condiciones de Trabajo (2004) Disponible en http://www.mtas.es/ insht/statistics/enct_5.htm

6. IV Encuesta Nacional de Condiciones de Trabajo (2001). Disponible en http://www.mtas.es/ insht/statistics/enct_4.htm
7. III Encuesta Nacional de Condiciones de Trabajo (1998). Disponible en http://www.mtas.es/ insht/statistics/enct_3.htm

8. Robb MJ \& Mansfield NJ. Self-reported musculoskeletal problems amongst professional truck drivers. Ergonomics 2007, Jun 50 (6): 814-827

9. Cui R, Tanigawa T, Sakurai S, Yamagishi K \& Iso H. Relationships between sleep-disordered breathing and blood pressure and excessive daytime sleepiness among truck drivers. Hypertens Res. 2006 Aug (8): 605-10.

10. S Heistaroa, P Jousilahtia, E Lahelmab, E Vartiainena \& P Puskaa. Self rated health and mortality: a long term prospective study in eastern Finland J Epidemiol Community Health 2001;55: 227-232.

11. Ordaz E, Maqueda, J, Olmedo O. Salud y Condiciones de Trabajo en el Transporte de Mercancías por Carretera. Madrid, Editado por ENMT nov. 2007 